\title{
Impact of dental cement on the peri-implant tissue Microbial comparison of two different cements in an in-vivo observational study
}

Korsch M., Marten S. M., Walther W., Vital M., Pieper D.H., Doetsch A.

Authors' affiliations:

Dr. Michael Korsch

- Dental Academy for Continuing Professional Development, Karlsruhe Lorenzstrasse 7, 76135 Karlsruhe, Germany

- $\quad$ Private practice, Center for Implantology and Oral Surgery, 69120 Heidelberg, Germany

- $\quad$ Clinic of Operative Dentistry, Periodontology and Preventive Dentistry, University Hospital, Saarland University, Building 73, 66421 Homburg, Germany

Prof. Dr. Winfried Walther

- Dental Academy for Continuing Professional Development, Karlsruhe Lorenzstrasse 7, 76135 Karlsruhe, Germany

Dr. Andreas Doetsch, Silke-Mareike Marten

- $\quad$ Institute of Functional Interfaces, Karlsruhe Institute of Technology (KIT), Karlsruhe, Germany

Prof. Dr. Dietmar H. Pieper, Dr. Marius Vital.

- Microbial Interactions and Processes Research Group, Helmholtz Centre for Infection Research, Braunschweig, Germany.

Corresponding author:

Dr. med. dent. Michael Korsch, M.A.

Dental Academy for Continuing Professional Development

Lorenzstrasse 7

76135 Karlsruhe

Germany

Phone: +49-721-9181-200

Fax: $\quad+49-721-9181-222$

E-mail: michael_korsch@za-karlsruhe.de

Author Contribution Statement:

Michael Korsch, Andreas Doetsch, Silke-Mareike Marten: Design, data collection, statistics, data analysis/interpretation, drafting article, approval of the submitted version. No conflict of interest.

Winfried Walther, Dietmar Pieper, Marius Vital: Design, statistics, drafting article, approval of the submitted version.

No conflict of interest. 


\begin{abstract}
Background

Undetected excess cement in cemented fixed implant-supported restorations involves the risk of periimplant inflammation. It is unclear what influence a revision of the suprastructure including the removal of existing excess cement will have on the peri-implant tissue and its microbial biofilm.
\end{abstract}

Purpose

The aim of the study was to investigate the impact of two different cements on the peri-implant biofilm.

Methods

In an observational study the suprastructures of 34 patients with cemented fixed implant-supported restorations were revised. In 20 patients a methacrylate cement (PIC) and in 14 patients a zinc oxide eugenol cement (TB) was used. After revision TB was used for recementation. During revision and follow-up one year after, microbial samples were obtained.

Results

Excess cement was found in $12(60 \%)$ of the 20 patients with PIC. Suppuration was observed in two (25\%) implants with PIC without excess cement (PIC-) and in all 12 (100\%) implants with PIC and excess cement (PIC+). Implants cemented with TB had neither excess cement nor suppuration. The taxonomic analysis of the microbial samples revealed an accumulation of oral pathogens in the PIC patients independent of the presence of excess cement. Significantly fewer oral pathogens occurred in patients with TB compared to patients with PIC. TB was used in all cases (PIC and TB) for recementation. In the follow-up check, suppuration was not found around any of the implants with PIC-, only around one implant with PIC+ and around one implant with TB. Bacterial species associated with severe periodontal infections that were abundant in PIC- and PIC+ samples before the revision were reduced after one year to levels found in the TB samples.

\title{
Conclusion
}

The revision of the suprastructures including the removal of excess cement and the recementation with TB have a distinct influence on the peri-implant tissue and its microbial biofilm.

Key words: dental cements, biofilms, molecular biological analysis, in-vivo observational study, implant 


\section{Introduction}

The cementation of fixed suprastructures on implants involves the risk of excess cement remaining in the peri-implant sulcus $\stackrel{1-3}{~}$. The prevalence of this complication is reported to amount to up to $60 \% \frac{4}{\text {. }}$. Excess cement favors the formation of a biofilm ${ }^{5}$ and may lead to peri-mucositis, peri-implantitis or even the loss of the implant if this complication remains undetected. The longer the time the excess cement has stayed in the peri-implant sulcus, the greater the risk of peri-implant inflammation $\stackrel{8}{\text {. The }}$

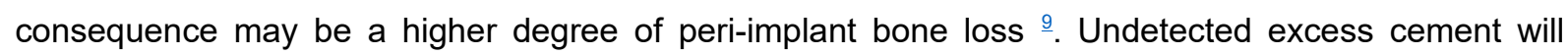
cause cement-associated peri-implant inflammation around approx. $80 \%$ of the implants .

For this reason many studies were made dealing with the avoidance of excess cement. One option is the modification of the dental restoration. Drainage holes in the abutment and suprastructure can reduce the amount of excess cement after cementation ${ }^{10-12}$. Other publications describe cementation protocols minimizing the risk of submucosal excess cement and recommend that during cementation the suprastructures should only partly, not completely, be filled with cement $\stackrel{2}{2}$. The temporary extraoral cementation on duplicate implant abutments can markedly reduce the risk of excess cement when the dental restoration is immediately inserted in the mouth before the cement has fully hardened. However, none of these protocols will eliminate the risk completely 15 .

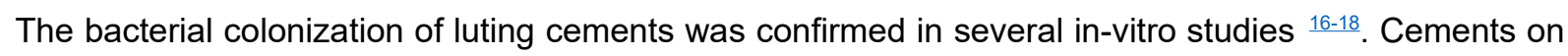
a methacrylate basis seem to be more prone to it than cements on a zinc oxide eugenol basis. In patients with cement-associated peri-implantitis the removal of excess cement from the sulcus will eliminate the peri-implantitis in about $75 \%$ of the cases. These studies, however, cover an observation period of not more than 3-4 weeks after cement removal. There are indications that certain cements will lead to peri-implant inflammation even in the absence of excess cement. It is therefore unclear what long-term influence the removal of excess cement will have on the peri-implant tissue. On the other hand, the instrumentation of the abutment using curettes and scalers may compromise its surface ${ }^{21}$. Roughening the abutment surface can itself again favor the formation of a biofilm $\underline{22}$.

Our hypothesis was that different types of cement have varying influence on the peri-implant biofilm. The aim of the present observational study was to investigate the effects of excess cement removal on the clinical findings and the microbial peri-implant biofilm.

\section{Material and methods}

Between April 2009 und February 2010, a methacrylate-based temporary cement (Premier Implant Cement (PIC), Premier ${ }^{\circledR}$ Dental Products Company, Plymouth Meeting, USA) was used for the fixation of suprastructures on implants. After some months several patients developed peri-implant inflammations which were connected with undetected excess cement. Due to these complications, a temporary cement based on zinc oxide eugenol (Temp Bond (TB), Kerr Sybron Dental Specialities, Glendora, USA) was used from March 2010. Both cements were not radiopaque at the time of the study.

In a clinical observational study, the influence of the type of cement used for fixed implant-supported restorations on the peri-implant tissue and the microbial biofilm was to be investigated. Patients' suprastructures that had been fixed with one of the two cements mentioned (PIC and TB) were revised 
between September and December 2013. In the revision the clinical findings were documented and microbial samples taken. As in some cases PIC had caused peri-implant infections and we had stopped using it in our clinic in March 2010, all suprastructures that had previously been fixed with PIC or TB were recemented with TB. In the clinical trial we were able to prove that PIC and TB differ in their effect on the peri-implant tissue and the submucosal biofilm. These results have already been published $\stackrel{5}{5}$. Implants with PIC-cemented suprastructures demonstrated significantly more peri-implant inflammations and oral pathogens than implants with TB-cemented suprastructures. What was unclear was whether, after revision and recementation with TB, the peri-implant tissue around implants with PIC will heal and a healthy microflora will again develop around such implants.

The present trial was made with the aim of investigating, in cases of cement-associated periimplantitis, the influence of revision and recementation with TB on the peri-implant tissue and the microbial biofilm. Thus, the patients whose suprastructures had been revised between September and December 2013 were followed up in the period from November 2014 to January 2015.

\section{Inclusion and exclusion criteria:}

Patients whose suprastructures were revised between September and December 2013 qualified for inclusion. All these patients had fixed implant-supported suprastructures cemented with either PIC or TB. At the time of revision the suprastructure needed to be in situ for at least 3.5 years. In addition, all these cases needed to be fully documented from the time of insertion of the dental restoration in the mouth. Suprastructures that demonstrated decementation after insertion of the dental restoration or after revision were excluded from the trial. All patients were fully informed in advance about the planned study and the clinical procedure. A signed Informed Consent Form was the condition for participation in the trial.

The institutional review board of the Medical Council of the Saarland examined and confirmed the project (approval was granted on November 24, 2014, reference no. 146/11).

\section{Clinical procedure of revision, documentation of clinical findings and sampling}

- Before the revision of the suprastructure, a pool sample was taken with sterile paper points from the peri-implant sulcus around each implant. Any existing signs of inflammation (pocket suppuration) were documented.

- At revision, the suprastructure was removed with dental forceps or a hook. In all cases the restorations could be removed without being damaged. After revision of the suprastructures including the abutments, the excess cement around each implant was documented.

- Finally, the peri-implant tissue was rinsed with chlorhexidine $0.12 \%$ and the suprastructure was recemented with TB cement.

Clinical procedure of follow-up, documentation of clinical findings and sampling

- A pool sample was taken with sterile paper points from the peri-implant sulcus around each implant. Any existing signs of inflammation (pocket suppuration) were documented.

- Finally, the peri-implant tissue was rinsed with chlorhexidine $0.12 \%$ 


\section{Study population (Fig. 1)}

Between January and May 2010, a total number of 38 patients had received prosthetic restoration using temporary methacrylate cement (PIC) or a zinc oxide eugenol cement (TB). The suprastructures were revised between September and December 2013. One year after revision therapy 34 patients could be followed up while the remaining 4 patients decided to opt out of the study.

PIC population:

This cohort included 20 patients (14 men, 6 women) aged between 19 and 77 years (average age 60.4 years) at the time of suprastructure cementation. The average retention time (period between cementation and revision) of the suprastructure was 4.1 years. In $12(60 \%)$ of these patients undetected excess cement was identified in the peri-implant tissue at revision. Based on this finding, the patients were subdivided according to the prevalence of excess cement.

Group 1 [PIC-] (PIC population without initially undetected excess cement):

This group included 8 patients ( 4 men, 4 women). The average age was 62.6 years.

Group 2 [PIC+] (PIC population with initially undetected excess cement):

This group included 12 patients (10 men, 2 women). The average age was 58.8 years.

\section{TB population:}

In this cohort there were 14 patients ( 7 men, 7 women). At the time of cementation the patients' age was between 34 and 72 years (average age 60.6 years). The average retention time of the suprastructure was 3.7 years. At the time of revision, undetected excess cement was not found in the peri-implant tissue of any of these patients. For this reason all 14 patients were combined in Group 3 [TB].

For the microbial analysis of the peri-implant tissue, bacterial samples were obtained by probing the peri-impant pocket of a total of 34 patients using sterile paper points and analyzed for taxonomic composition by sequencing the V1-V2 variable regions of the 16S rRNA gene.

\section{Microbial methods}

DNA extraction

Paper point tips were cut and placed in sterile $1.5 \mathrm{~mL}$ tubes, covered with $1 \mathrm{~mL}$ nuclease free water (Life technologies, Carlsbad, CA) and vortexed for $10 \mathrm{~s}$. For debonding of biofilm microbes, the samples were treated for 2:30 h on a thermomixer (Eppendorf Instruments, Hamburg, Germany) at 22 
${ }^{\circ} \mathrm{C}$ and $1400 \mathrm{rpm}$, followed by $10 \mathrm{~s}$ vortexing, $5 \mathrm{~min}$ sonication at $43 \mathrm{kHz}$ and again vortexed for $10 \mathrm{~s}$. The supernatants were collected and centrifuged for $10 \mathrm{~min}$ at 13,300 rpm (Biofuge Pico, Heraeus). All supernatants were discarded and the pellets stored for $24 \mathrm{~h}$ at $-80^{\circ} \mathrm{C}$. The collected pellets of the supernatant were treated with $180 \mu \mathrm{L}$ lysozyme solution $(20 \mathrm{mg} / \mathrm{mL}$, SIGMA-Aldrich, Taufkirchen, Germany; $20 \mathrm{mM}$ Tris- $\mathrm{HCl}$, pH 8.0, $2 \mathrm{mM}$ EDTA, $1.2 \%$ Triton) under shaking at $37^{\circ} \mathrm{C}$ for $2: 15 \mathrm{~h}$, followed by proteinase $K$ digestion ( $20 \mu \mathrm{L}$ proteinase $\mathrm{K}$ and $200 \mu \mathrm{L}$ buffer $\mathrm{AL}$ ) for 1:30 $\mathrm{h}$ while shaking at $56^{\circ} \mathrm{C}$. DNA was extracted using commercial extraction protocols for genomic DNA (QIAamp Mini Kit, Qiagen, Hilden, Germany). Finally, the DNA was eluted with $100 \mu \mathrm{L}$ PCR-clean water and the concentration was quantified using the NanoDrop equipment (PEQLAB, Erlangen, Germany).

\section{Amplicon library preparation and taxonomic analysis of pathogen groups}

Previously described primers for the V1 and V2 region of the 16S rRNA gene (27F and 338R) were used for amplification and libraries prepared as previously described. Illumina sequencing and bioinformatic processing of paired end reads ( $250 \mathrm{bp}$ ) was done as previously described with modifications ${ }^{23}$. Raw reads were merged and subsequently aligned using MOTHUR (gotoh algorithm using the SILVA reference database) prior to pre-clustering (allowing for 2 mismatches) $\underline{25} \underline{26}$ Phylotypes exhibiting an average abundance of $\geq 0.005 \%$ and a sequence length of $\geq 250 \mathrm{bp}$ were considered for follow-up analysis.

A total number of 996 different phylotypes (OTUs) remained. Relative percent abundances were used for further analysis. Phylotypes were assigned to a taxonomic affiliation based on the naïve Bayesian classification ${ }^{27}$ with a threshold of $80 \%$ followed by manual curation as previously described $\stackrel{23}{ }$. To identify phylotypes indicating the presence of the three species of the red complex as defined by Socransky and Haffajee $\underline{28}$, i.e., Porphyromonas gingivalis, Treponema denticola and Tannerella forsythia, sequences of all isolates of the respective genera available from the RDP server (http://rdp.cme.msu.edu) were separately downloaded. Those sequences covering the 16S rRNA region analyzed here were trimmed and aligned with amplicon sequences where the Bayesian classification has indicated that they derived from members of the respective genus. Sequences were aligned using MUSCLE $\stackrel{29}{2}$ and phylogenetic analyses conducted in MEGA7 $\stackrel{30}{2}$. The evolutionary histories of genes were inferred using the neighbor-joining method and the Jukes-Cantor model. All positions containing alignment gaps and missing data were eliminated only in pairwise sequence comparisons. Amplicon sequences were then assigned to originate from a previously described species if they are localized in a branch supported by high bootstrap values $(>80)$ formed exclusively by members of that species.

\section{Statistical methods}

The data were analyzed with IBM SPSS Statistics 21 (IBM SPSS Statistics, IBM, Armonk, NY, USA). The difference in excess cement between PIC and TB was analyzed using the Chi-squared test for statistical independence. The differences in the relative abundance of pathogen groups before and after the cement replacement were analyzed using the Wilcoxon signed rank test, a non-parametrical 
test of the null hypothesis that the median of the distribution of differences is zero. The resulting Pvalues were corrected for multiple testing by using the Benjamini-Hochberg method $\stackrel{31}{2}$. A principal component analysis of the relative abundances was performed using a singular value decomposition algorithm with centering of the data. The Wilcoxon signed rank test and the principal component analysis were performed using the signrank and pca functions of the Matlab Statistics toolbox R2015a (Mathworks Inc., Natick, MA, USA).

\section{Results}

The two populations PIC and TB were homogeneous in terms of gender distribution, average age and oral retention times of the suprastructures, since no significant differences of these factors were found (ANOVA test). At the time of revision two implants $(25 \%)$ in the PIC- group and all twelve implants $(100 \%)$ in the PIC+ group were affected by suppuration. In the TB group none of the 14 implants had this complication. The difference between the three groups was significant (Chi-squared with $2 \mathrm{df}$, $27,807 ; p<0.001)$. The follow-up one year after revision presented a completely different clinical picture. In the PIC- group there was no implant with suppuration any more, and in the PIC+ group there was just one such implant. However, one implant in the TB group also suppurated. The difference between the three groups was no longer significant.

\section{Results of the microbial evaluation}

The taxonomic analysis based on amplicons of the gene encoding the $16 \mathrm{~S}$ ribosomal subunit identified 996 individual phylotypes that were assigned to 122 different genera using the RDP classifier. The subgingival microbiota across all samples in this study is dominated by the six phyla Actinobacteria, Bacteroidetes, Firmicutes, Fusobacteria, Proteobacteria and Spirochaetes (Fig. 2). Notably, the different groups of cement types showed a much more similar taxonomic composition after the replacement of the cements by TB (Fig. 2, lower panels). Before the replacement, the subgingival microbiota of implants with PIC cement (PIC- and PIC+) contained larger fractions of Bacteroidetes and Spirochaetes. These two phyla include the three species that are classified as the "red complex" in the Socransky nomenclature $\stackrel{28}{ }$, namely Porphyromonas gingivalis, Tannerella forsythia (both Bacteroidetes) and Treponema denticola (Spirochaetes). The red complex bacteria have been

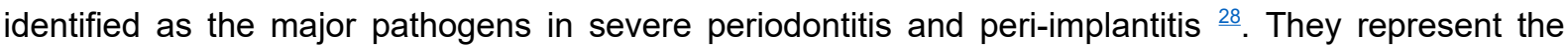
majority of Bacteroidetes and Spirochaetes sequences in the PIC cement samples. An individual comparison of the relative abundance of the three red complex species in each of the different sample groups confirms that the previously observed higher relative abundance of red complex species ${ }^{5}$ was directly related to the PIC cement and reverted back to values similar to TB cement samples after PIC cement was replaced by TB cement (Fig. 3 A and B ${ }^{5}$ ). Interestingly, three implants with TB cement exhibited an abundance of $\underline{P}$. gingivalis that was much higher than in other implants of this group, which remained at a high level albeit with some reduction even after the replacement of the cement by fresh TB. In at least one of these cases the detection of $\underline{P}$. gingivalis can be related to a documented periodontal infection and these results might therefore be unrelated to the cement type. 
A principal component analysis of all samples in the study was performed (Fig. 4) in analogy to the previous study, resulting in a similar pattern with three main "branches" of data points. Roughly, these three groups of patients are characterized by a subgingival microbiome dominated by commensal oral bacteria (lower branch in Fig. 4), Fusobacterium nucleatum (right branch) or bacteria of the Socransky red complex (upper branch). In accordance with the reduction of the Socransky red complex after implant revision and replacement of the cement, the latter group only contains data points reflecting implants (mostly of the PIC-ex and PIC+ex groups) before the revision.

\section{Discussion}

The clinical picture of the TB population and the PIC population differed very clearly at the time of revision. After an average retention period of 4.1 years, excess cement left in the sulcus caused periimplant suppuration in all cases, although 2 implants (25\%) without excess cement for which PIC had been used (PIC-) were also affected by suppuration. None of the implants with TB-cemented suprastructures had excess cement or suppuration. It is to be assumed that cementation with zinc oxide eugenol cement can also lead to excess cement $\stackrel{32}{ }$, which, however, will apparently dissolve in contact with the sulcus fluid in the peri-implant tissue. In-vitro studies confirmed this assumption and could prove that, in contact with artificial saliva, zinc oxide eugenol cements are showing signs of disintegration after a short period already $\underline{33}$.

At follow-up one year after revision, the clinical picture of the PIC population had completely changed. Only one implant of this population still suppurated. It is to be assumed that both the revision of the dental restorations including the removal of the excess cement and the recementation with TB had an essential influence on the clinical findings. We know for sure that the two principal components, i.e. zinc oxide and eugenol, in TB cement have an effect on the peri-implant biofilm. Zinc oxide is said to have an antimicrobial activity. Eugenol, an isolated compound of cloves oil (Syzygium aromaticum), also has an antimicrobial effect as have many essential oils. Eugenol is able to inhibit the growth of a large number of microorganisms without being able to destroy them. It has an antibacterial, antiviral as well as an antifungal effect $\stackrel{40-43}{ }$. The clinical findings after a follow-up period of one year may be assumed to be stable and the result of removing the excess cement and changing the type of cement (from PIC to TB) when the dental restorations were recemented in the revision treatment.

The oral microbiome is extremely diverse and large numbers of different taxa have been described in the literature ${ }^{44-46}$. The subgingival plaque has been described as being dominated by bacterial species belonging mainly to five to six phyla $\stackrel{46}{a}$ that were also detected in this study (Fig. 2). The principal component analysis demonstrated that there is substantial variance across all samples that is only partially covered by our analysis (only $\sim 30 \%$ of the total variance is explained by the first two principal components presented in Fig. 4). A large proportion of this variance might be influenced by factors that are beyond the scope of this study and the available additional metadata (including smoking, recent antibiosis and diabetes), could not be clearly correlated with the data (data not shown). Nevertheless, the results supported the connection between the abundance of pathogenic bacteria with the presence of PIC and undetected excess cement. In the initial study of implants with the different cement types, a shift was observed towards a higher relative abundance of bacteria belonging to the Socransky red complex within the subgingival plaque that was specific to the group of implants with PIC cement. 
Comparing the taxonomic results for these implants one year after the replacement clearly shows that the microbiome has reverted to a state that closely resembles the composition of implants with TB cement (before and after revision) and is also in line with the subgingival microbiome published elsewhere. This observation clearly confirms that the red complex bacteria are specifically more abundant when PIC cement is used and that the risk for an infection of the peri-implant tissue can be strongly reduced by avoiding this cement type and replacing it where it has already been applied. The results could be influenced by the small group size which is a limitation of the study.

Thus, implants whose suprastructures were fixed with a methacrylate-based cement apparently have more oral pathogenic bacteria in the peri-implant sulcus than implants that were cemented with zinc oxide eugenol cement. In this respect the material properties of zinc oxide eugenol are perfectly suitable for the permanent cementation of fixed implant-supported dental restorations. Zinc oxide eugenol cement, however, is stated to be a temporary luting cement so that its use as a permanent luting material is not in agreement with the product description. Compared to harder cements, its weaker retentive properties, however, do not seem to be of clinical relevance ${ }^{47-49}$. Therefore the permanent cementation of fixed implant-supported dental suprastructures with zinc oxide eugenol cement is to be recommended.

\section{Acknowledgements}

We thank Tamara Strobl for support during sample and data collection. Dietmar H. Pieper and Marius Vital were supported by iMed, the Helmholtz Association's Initiative on Personalized Medicine.

\section{References}

1. Callan DP, Cobb CM. Excess cement and peri-implant disease. JIACD 2009; 1: 61-68. 
2. Dumbrigue HB, Abanomi AA, Cheng LL. Techniques to minimize excess luting agent in cement-retained implant restorations. J Prosthet Dent 2002; 87: 112-114.

3. Gapski R, Neugeboren N, Pomeranz AZ, Reissner MW. Endosseous implant failure influenced by crown cementation: a clinical case report. Int J Oral Maxillofac Implants 2008; 23: 943-946.

4. Korsch M, Obst U, Walther W. Cement-associated peri-implantitis: a retrospective clinical observational study of fixed implant-supported restorations using a methacrylate cement. Clin Oral Implants Res 2014; 25: 797-802.

5. Korsch M, Marten SM, Dotsch A, Jauregui R, Pieper DH, Obst U. Effect of dental cements on peri-implant microbial community: comparison of the microbial communities inhabiting the periimplant tissue when using different luting cements. Clin Oral Implants Res 2016; 27: e161-e166.

6. Pauletto N, Lahiffe BJ, Walton JN. Complications associated with excess cement around crowns on osseointegrated implants: a clinical report. Int J Oral Maxillofac Implants 1999; 14: 865868.

7. Wilson TG, Jr. The positive relationship between excess cement and peri-implant disease: a prospective clinical endoscopic study. J Periodontol 2009; 80: 1388-1392.

8. Korsch M, Robra BP, Walther W. Cement-Associated Signs of Inflammation: Retrospective Analysis of the Effect of Excess Cement on Peri-implant Tissue. Int J Prosthodont 2015; 28: 11-18.

9. Linkevicius T, Puisys A, Vindasiute E, Linkeviciene L, Apse P. Does residual cement around implant-supported restorations cause peri-implant disease? A retrospective case analysis. Clin Oral Implants Res 2013; 24: 1179-1184.

10. Patel D, Invest JC, Tredwin CJ, Setchell DJ, Moles DR. An analysis of the effect of a vent hole on excess cement expressed at the crown-abutment margin for cement-retained implant crowns. $J$ Prosthodont 2009; 18: 54-59.

11. Wadhwani C, Hess T, Pineyro A, Chung KH. Effects of abutment and screw access channel modification on dislodgement of cement-retained implant-supported restorations. Int J Prosthodont 2013; 26: 54-56.

12. Wadhwani $\mathrm{C}$, Pineyro A, Hess T, Zhang H, Chung KH. Effect of implant abutment modification on the extrusion of excess cement at the crown-abutment margin for cement-retained implant restorations. Int J Oral Maxillofac Implants 2011; 26: 1241-1246.

13. Wadhwani $C$, Pineyro A. Technique for controlling the cement for an implant crown. $J$ Prosthet Dent 2009; 102: 57-58.

14. Yuzbasioglu E. A modified technique for extraoral cementation of implant retained restorations for preventing excess cement around the margins. J Adv Prosthodont 2014; 6: 146-149.

15. Frisch E, Ratka-Kruger P, WeigI P, Woelber J. Minimizing Excess Cement in Implant-Supported Fixed Restorations Using an Extraoral Replica Technique: A Prospective 1-Year Study. Int J Oral Maxillofac Implants 2015; 30: 1355-1361.

16. Winkler C, Schafer L, Felthaus O, Allerdings J, Hahnel S, Behr M, Burgers R. The bacterial adhesion on and the cytotoxicity of various dental cements used for implant-supported fixed restorations. Acta Odontol Scand 2014; 72: 241-250.

17. Velazquez-Enriquez U, Scougall-Vilchis RJ, Contreras-Bulnes R, Flores-Estrada J, Uematsu S, Yamaguchi R. Quantitative analysis of S. mutans and S. sobrinus cultivated independently and adhered to polished orthodontic composite resins. J Appl Oral Sci 2012; 20: 544-549.

18. Raval NC, Wadhwani CP, Jain S, Darveau RP. The Interaction of Implant Luting Cements and Oral Bacteria Linked to Peri-Implant Disease: An In Vitro Analysis of Planktonic and Biofilm Growth--A Preliminary Study. Clin Implant Dent Relat Res 2015; 17: 1029-1035.

19. Obst U, Marten SM, Niessner C, Hartwig E, Korsch M, Walther W. Diversity of patients microflora on orthopaedic and dental implants. Int J Artif Organs 2012; 35: 727-734.

20. Korsch M, Walther W. Peri-Implantitis Associated with Type of Cement: A Retrospective Analysis of Different Types of Cement and Their Clinical Correlation to the Peri-Implant Tissue. Clin Implant Dent Relat Res 2015; 17 Suppl 2: e434-443.

21. Agar JR, Cameron SM, Hughbanks JC, Parker MH. Cement removal from restorations luted to titanium abutments with simulated subgingival margins. The Journal of Prosthetic Dentistry 1997; 78: 43-47. 
22. Dmytryk JJ, Fox SC, Moriarty JD. The effects of scaling titanium implant surfaces with metal and plastic instruments on cell attachment. J Periodontol 1990; 61: 491-496.

23. Camarinha-Silva A, Jauregui R, Chaves-Moreno D, Oxley AP, Schaumburg F, Becker K, WosOxley ML, Pieper DH. Comparing the anterior nare bacterial community of two discrete human populations using Illumina amplicon sequencing. Environ Microbiol 2014; 16: 2939-2952.

24. Chaves-Moreno D, Plumeier I, KahI S, Krismer B, Peschel A, Oxley AP, Jauregui R, Pieper DH. The microbial community structure of the cotton rat nose. Environ Microbiol Rep 2015; 7: 929-935. 25. Cole JR, Wang Q, Fish JA, Chai B, McGarrell DM, Sun Y, Brown CT, Porras-Alfaro A, Kuske CR, Tiedje JM. Ribosomal Database Project: data and tools for high throughput rRNA analysis. Nucleic Acids Res 2014; 42: D633-642.

26. Schloss PD, Westcott SL, Ryabin T, Hall JR, Hartmann M, Hollister EB, Lesniewski RA, Oakley BB, Parks DH, Robinson CJ, Sahl JW, Stres B, Thallinger GG, Van Horn DJ, Weber CF. Introducing mothur: open-source, platform-independent, community-supported software for describing and comparing microbial communities. Appl Environ Microbiol 2009; 75: 7537-7541.

27. Wang Q, Garrity GM, Tiedje JM, Cole JR. Naive Bayesian classifier for rapid assignment of rRNA sequences into the new bacterial taxonomy. Appl Environ Microbiol 2007; 73: 5261-5267.

28. Socransky SS, Haffajee AD. Periodontal microbial ecology. Periodontol 2000 2005; 38: 135187.

29. Edgar RC. MUSCLE: multiple sequence alignment with high accuracy and high throughput. Nucleic Acids Res 2004; 32: 1792-1797.

30. Kumar S, Stecher G, Tamura K. MEGA7: Molecular Evolutionary Genetics Analysis Version 7.0 for Bigger Datasets. Mol Biol Evol 2016; 33: 1870-1874.

31. Benjamini $Y$, Hochberg Y. Controlling the false discovery rate: a practical and powerful approach to multiple testing. J Roy Stat Soc B 1995; 57: 289-300.

32. Linkevicius $T$, Vindasiute $E$, Puisys $A$, Peciuliene $V$. The influence of margin location on the amount of undetected cement excess after delivery of cement-retained implant restorations. Clin Oral Implants Res 2011; 22: 1379-1384.

33. Yanikoglu N, Yesil Duymus Z. Evaluation of the solubility of dental cements in artificial saliva of different pH values. Dent Mater J 2007; 26: 62-67.

34. Xie $\mathrm{Y}, \mathrm{He} \mathrm{Y}$, Irwin PL, Jin T, Shi X. Antibacterial activity and mechanism of action of zinc oxide nanoparticles against Campylobacter jejuni. Appl Environ Microbiol 2011; 77: 2325-2331.

35. Jin T, Sun D, Su JY, Zhang H, Sue HJ. Antimicrobial efficacy of zinc oxide quantum dots against Listeria monocytogenes, Salmonella Enteritidis, and Escherichia coli O157:H7. J Food Sci 2009; 74: 4652.

36. Freires IA, Bueno-Silva B, Galvao LC, Duarte MC, Sartoratto A, Figueira GM, de Alencar SM, Rosalen PL. The Effect of Essential Oils and Bioactive Fractions on Streptococcus mutans and Candida albicans Biofilms: A Confocal Analysis. Evid Based Complement Alternat Med 2015; 2015: 871316.

37. Jeon JG, Rosalen PL, Falsetta ML, Koo H. Natural products in caries research: current (limited) knowledge, challenges and future perspective. Caries Res 2011; 45: 243-263.

38. Shapiro S, Meier A, Guggenheim B. The antimicrobial activity of essential oils and essential oil components towards oral bacteria. Oral Microbiol Immunol 1994; 9: 202-208.

39. Perugini Biasi-Garbin R, Saori Otaguiri E, Morey AT, Fernandes da Silva M, Belotto Morguette AE, Armando Contreras Lancheros C, Kian D, Perugini MR, Nakazato G, Duran N, Nakamura CV, Yamauchi LM, Yamada-Ogatta SF. Effect of Eugenol against Streptococcus agalactiae and Synergistic Interaction with Biologically Produced Silver Nanoparticles. Evid Based Complement Alternat Med 2015; 2015: 861497.

40. Feres M, Figueiredo LC, Barreto IM, Coelho MH, Araujo MW, Cortelli SC. In vitro antimicrobial activity of plant extracts and propolis in saliva samples of healthy and periodontally-involved subjects. J Int Acad Periodontol 2005; 7: 90-96.

41. Burt SA, Reinders RD. Antibacterial activity of selected plant essential oils against Escherichia coli 0157:H7. Lett Appl Microbiol 2003; 36: 162-167. 
42. da Silva JK, Silva JR, Nascimento SB, da Luz SF, Meireles EN, Alves CN, Ramos AR, Maia JG. Antifungal activity and computational study of constituents from Piper divaricatum essential oil against Fusarium infection in black pepper. Molecules 2014; 19: 17926-17942.

43. Astani A, Reichling J, Schnitzler P. Screening for antiviral activities of isolated compounds from essential oils. Evid Based Complement Alternat Med 2011; 2011: 253643.

44. Bik EM, Long CD, Armitage GC, Loomer P, Emerson J, Mongodin EF, Nelson KE, Gill SR, FraserLiggett CM, Relman DA. Bacterial diversity in the oral cavity of 10 healthy individuals. ISME J 2010; 4: 962-974.

45. Dewhirst FE, Chen T, Izard J, Paster BJ, Tanner AC, Yu WH, Lakshmanan A, Wade WG. The human oral microbiome. J Bacteriol 2010; 192: 5002-5017.

46. Segata N, Haake SK, Mannon P, Lemon KP, Waldron L, Gevers D, Huttenhower C, Izard J. Composition of the adult digestive tract bacterial microbiome based on seven mouth surfaces, tonsils, throat and stool samples. Genome Biol 2012; 13: R42.

47. Nissan J, Narobai D, Gross O, Ghelfan O, Chaushu G. Long-term outcome of cemented versus screw-retained implant-supported partial restorations. Int J Oral Maxillofac Implants 2011; 26: 11021107.

48. Frisch E, Ziebolz D, Rinke S. Long-term results of implant-supported over-dentures retained by double crowns: a practice-based retrospective study after minimally 10 years follow-up. Clin Oral Implants Res 2013; 24: 1281-1287.

49. Korsch $M$, Walther W. Retrospective analysis of loosening of cement-retained vs screwretained fixed implant-supported reconstructions. Quintessence Int 2015; 46: 583-589. 
Fig. 1: The flow chart shows the patients who were treated with TB and PIC from April 2009 to February 2010. The blue boxes are the patients that were followed-up for the purpose of the present study. The blue boxes represent the 3 groups investigated in the study.

\section{Fig. 2: Average taxonomical composition of the subgingival microbiota}

The pie graphs present relative abundances of bacterial phyla averaged across all samples of the three sample groups TB (Temp Bond cement), PIC- (Premier Implant cement without excess cement), $\mathrm{PIC}+$ (Premier Implant cement with excess cement). Results are shown for the samples before and after the implant revision and replacement of the cement. Different colors correspond to different bacterial phyla as indicated in the color legend. The contribution of the three species of the Socransky red complex ${ }^{28}$ are highlighted in red.

\section{Fig. 3: Relative abundance of the Socransky red complex species before and after cement} replacement

A) Boxplots of the relative abundances of each of the three species belonging to the Socransky red complex. Samples were grouped by the type of cement and observed formation of excess cement (TB - Temp Bond cement, PIC- - Premier Implant cement without excess cement, PIC+ - Premier Implant cement with excess cement) and time of sampling (1 - before each cement was replaced by fresh Temp Bond cement, 2 - after replacement). B) Boxplots of the differences in relative species abundance before and after implant revision and replacement of the cement. The differences were calculated for each patient sample individually and the boxplots show the distribution of differences for each cement type. An asterisk $\left({ }^{*}\right)$ indicates that the median of the differences is significantly different from zero ( $p<0.05$, Wilcoxon signed rank test, corrected for multiple testing).

\section{Fig. 4: Principal component analysis of relative abundances.}

Each circle represents the taxonomic composition of an individual sample. Different colors indicate the different groups of samples: TB - Temp Bond cement, PIC- - Premier Implant cement without excess cement, PIC+ - Premier Implant cement with excess cement. Samples were obtained before (empty circles) and after (filled circles) the cement was replaced by fresh TB cement. For each principal component, the percentage of total variance explained by this principal component is given in parenthesis. Arrows indicate the loadings of the nine phylotypes with the largest loadings in the first two principal components, i.e. those phylotypes that contributed most to the position of data points in this plot. If only a genus name is given, the annotation of the species name was below the confidence threshold. 